\title{
SEASONAL ADJUSTMENT AND THE BUSINESS CYCLE IN UNEMPLOYMENT
}

\author{
Philip Hans FRANSES* \\ Econometric Institute \\ Erasmus University Rotterdam \\ and \\ Paul DE BRUIN \\ Tinbergen Institute \\ University of Amsterdam
}

May 1999

Econometric Institute Report 9923/A

\begin{abstract}
Several recent studies show that seasonal variation and cyclical variation in unemployment are correlated. A common finding is that seasonality tends to differ across the business cycle stages of recessions and expansions. Since seasonal adjustment methods assume that the two sources of variation can somehow be separated, the present study examines the impact of seasonal adjustment on the analysis of cyclical patterns. Seasonally adjusted quarterly unemployment data for 5 G-7 countries are modeled by a Smooth Transition Autoregression [STAR] while the corresponding unadjusted data are modeled by a so-called Seasonal STAR [SEASTAR]. A comparison of the implied estimated peaks and troughs shows that there is substantial agreement on the business cycle chronologies, albeit that for seasonally adjusted data recessionary periods tend to last longer.

Key words: Unemployment, seasonality, business cycle, seasonal adjustment JEL classification: C22, E24

\footnotetext{
${ }^{*}$ Correspondence to: Econometric Institute, Erasmus University Rotterdam, P.O. Box 1738, NL-3000 DR Rotterdam, The Netherlands, fax: +3110 4527746 (e-mail: franses@few.eur.nl). We thank the Special Issue Editor and seminar participants of the Institute of Advanced Studies in Vienna and the Tinbergen Institute in Amsterdam for their suggestions.
} 


\section{INTRODUCTION}

Additional to (nonlinear) business cycle features, most quarterly unemployment data display pronounced seasonal variation. This aspect renders a substantial interest in designing seasonal adjustment methods. A key assumption for most such methods is that seasonal variation is (approximately) uncorrelated with cyclical variation. Recent empirical studies, however, have questioned this assumption. For example, Canova and Ghysels (1994) and Franses (1995) show that seasonality in unemployment (and other quarterly macroeconomic variables) tends to change with the business cycle. Miron (1996) arrives at essentially the same qualitative conclusion using alternative methods.

When business cycle variation and seasonal variation are dependent, it is of interest to study the consequences of applying seasonal adjustment methods. Ooms and Franses (1997) show for US and German unemployment that seasonally adjusted [SA] data can convey too optimistic views on the economy in specific quarters when in fact the economy enters a recession. Hence the interpretation of seasonally adjusted data can become difficult. With respect to modeling SA data, Ghysels and Perron (1993) show that seasonal adjustment can smooth away level shifts in the data. This may be interpreted as that SA data can display less obvious nonlinear features. Finally, Franses and Paap (1999) show using Monte Carlo simulations and empirical examples that inference from a Markov switching regime model differs across SA and not SA [NSA] data, but that the nonlinear feature does not disappear. Briefly, the latter authors find that one may arrive at different business cycle chronologies for SA and NSA data.

In this paper we aim to add to the findings in Franses and Paap (1999) by considering nonlinear time series models for quarterly SA and NSA unemployment series. To be able to arrive at generalizing statements, we consider data for the G-7 countries, where we find evidence for STAR type nonlinearity for 5 of the 7 countries. Also, we will construct models which are approximately the same across all variables. The SA data will be described by a smooth transition autoregression [STAR], see Granger and Teräsvirta (1993), and the NSA data are analyzed using a so-called seasonal STAR [SEASTAR], introduced in Franses (1998) and analyzed in detail in Franses and de Bruin (1999). The transition functions in both models will be compared, and the implied business cycle chronologies will be evaluated.

The outline of this paper is as follows. In Section 2, we discuss the econometric models 
for SA and NSA data. In Section 3, we compare the empirical results for country-specific time series. The main result is that the peaks and troughs generally show moderate to substantial agreement on the business cycle chronologies, and that for seasonally adjusted data recessionary periods tend to last longer. Some conclusions are given in Section 4.

\section{ECONOMETRIC MODELING}

This section deals with a general outline of the econometric modeling approach taken in this study. Denote quarterly seasonally unadjusted unemployment (number of unemployed or rate) as $y_{t}$, and denote the corresponding adjusted variable as $y_{t}^{a}$. In Sections 2.1 and 2.2, we discuss modeling $y_{t}$ and $y_{t}^{a}$, respectively. In Section 2.3 , we elaborate on the method to compare inference on business cycle variation for both models. Notice that $y_{t}$ and $y_{t}^{a}$ are logged series in case the data concern the number of unemployed, and that they are left untouched when they concern unemployment rates.

\subsection{SEASTAR models for seasonally unadjusted data}

Most quarterly observed unemployment data for industrialized countries, measured during the last two or three decades, show an upward trend. As it is unlikely that this trend is deterministic, it is commonly assumed that first differencing the data yields a time series which can be usefully analyzed. Denote this time series as $x_{t}=y_{t}-y_{t-1}$. Furthermore, denote $D_{s, t}, s=1,2,3,4$ as the usual seasonal dummy variables with $D_{s, t}=1$ when $t$ corresponds with season $s$, and $D_{s, t}=0$ otherwise. Finally, denote $z_{t}$ as the switching variable (or indicator variable), which determines the business cycle regimes. When $z_{t}$ takes a value above a certain threshold, the economy is said here to enter a recession. This is because we will assume that $z_{t}$ is a function of previous unemployment.

The seasonal STAR [SEASTAR] model put forward in Franses (1998) is given by

$$
\begin{aligned}
x_{t}= & F S\left(z_{t-d}\right)\left(\delta_{1}^{R} D_{1, t}+\delta_{2}^{R} D_{2, t}+\delta_{3}^{R} D_{3, t}+\delta_{4}^{R} D_{4, t}\right) \\
& +\left[1-F S\left(z_{t-d}\right)\right]\left(\delta_{1}^{E} D_{1, t}+\delta_{2}^{E} D_{2, t}+\delta_{3}^{E} D_{3, t}+\delta_{4}^{E} D_{4, t}\right) \\
& +F C\left(z_{t-d}\right)\left(\phi_{1}^{R} x_{t-1}+\phi_{2}^{R} x_{t-2}+\ldots+\phi_{p}^{R} x_{t-p}\right) \\
& +\left[1-F C\left(z_{t-d}\right)\right]\left(\phi_{1}^{E} x_{t-1}+\phi_{2}^{E} x_{t-2}+\ldots+\phi_{p}^{E} x_{t-p}\right)+\epsilon_{t},
\end{aligned}
$$


where $\epsilon_{t}$ denotes a zero mean white noise time series with variance $\sigma_{\epsilon}^{2}$. The two transition functions $F S($.$) for seasonal variation and F C($.$) for the business cycle are here defined by$

$$
F S\left(z_{t-d}\right)=\left\{1+\exp \left[-\gamma_{s}\left(z_{t-d}-\mu_{s}\right)\right]\right\}^{-1}, \gamma_{s}>0
$$

and

$$
F C\left(z_{t-d}\right)=\left\{1+\exp \left[-\gamma_{c}\left(z_{t-d}-\mu_{c}\right)\right]\right\}^{-1}, \gamma_{c}>0 .
$$

When $z_{t-d}$ (which is a function of past unemployment) exceeds the thresholds $\mu_{s}$ and $\mu_{c}$, the transition functions take values that approach unity. Hence, in case $F S\left(z_{t-d}\right)$ and $F C\left(z_{t-d}\right)$

approach 1 , the observation at time $t$ is said to correspond with a recession $(R)$, and when they approach 0 , the data correspond with an expansion $(E)$ (which explains the sub-indices for the parameters).

The model (1)-(3) can be viewed as a restriction on a very general SEASTAR model, see Franses and de Bruin (1999). In fact, the transition variable $z_{t}$ can differ across $F S$ and $F C$, as well as the delay parameter $d$. Empirical evidence in Franses (1998), however, indicates that the restricted model fits many unemployment data quite well.

As the SEASTAR contains two nonlinear switching functions, it is of importance to test for SEASTAR type nonlinearity before considering parameter estimation. Tests can be based on the auxiliary regression

$$
\begin{aligned}
x_{t}= & \sum_{s=1}^{4} \delta_{s} D_{s, t}+\sum_{s=1}^{4} \delta_{s, 1} D_{s, t} z_{t-d}+\sum_{s=1}^{4} \delta_{s, 2} D_{s, t} z_{t-d}^{2}+\sum_{s=1}^{4} \delta_{s, 3} D_{s, t} z_{t-d}^{3} \\
& +\sum_{i=1}^{p} \phi_{i} x_{t-i}+\sum_{i=1}^{p} \phi_{i, 1} x_{t-i} z_{t-d}+\sum_{i=1}^{p} \phi_{i, 2} x_{t-i} z_{t-d}^{2}+\sum_{i=1}^{p} \phi_{i, 3} x_{t-i} z_{t-d}^{3}+u_{t} .
\end{aligned}
$$

When the $\chi^{2}$ test statistic for $\delta_{s, 1}=\delta_{s, 2}=\delta_{s, 3}=0$ is significant, one should include the $F S$ function, and when it is significant for $\phi_{i, 1}=\phi_{i, 2}=\phi_{i, 3}=0$, one should include the $F C$ function. In our empirical application we follow the preliminary results in Franses (1998) and set $d=1$.

\subsection{STAR models for seasonally adjusted data}

If (1) with (2)-(3) yield good descriptions of the seasonally unadjusted data, it is not unlikely that the seasonally adjusted data can also be described by a STAR model. This is substantiated by simulation evidence in Franses and de Bruin (1999). Again, the adjusted data are 
first differenced, that is, models are constructed for $x_{t}^{a}=y_{t}^{a}-y_{t-1}^{a}$. In the absence of seasonal fluctuations, a STAR model for $x_{t}^{a}$ may be given by

$$
\begin{aligned}
x_{t}^{a}= & F\left(z_{t-m}^{a}\right)\left(\delta^{R}+\alpha_{1}^{R} x_{t-1}^{a}+\alpha_{2}^{R} x_{t-2}^{a}+\ldots+\alpha_{k}^{R} x_{t-k}^{a}\right) \\
& +\left[1-F\left(z_{t-m}^{a}\right)\right]\left(\delta^{E}+\alpha_{1}^{E} x_{t-1}^{a}+\alpha_{2}^{E} x_{t-2}^{a}+\ldots+\alpha_{k}^{E} x_{t-k}^{a}\right)+\nu_{t},
\end{aligned}
$$

where $\nu_{t}$ denotes a white noise time series with variance $\sigma_{\nu}^{2}$. The transition function is defined by

$$
F\left(z_{t-m}^{a}\right)=\left\{1+\exp \left[-\gamma\left(z_{t-m}^{a}-\mu\right)\right]\right\}^{-1}, \gamma>0
$$

where $z_{t-m}^{a}$ is the transition variable, which is a function of past seasonally adjusted (changes)

in unemployment. Again, the parameters in expansions are marked with an $E$, and those in recessions with an $R$. To test for STAR type nonlinearity, we consider a similar test as in (4), where we set $m=1$.

\subsection{Comparing results from SEASTAR and STAR models}

In this paper we estimate the parameters of (1)-(3) and (5)-(6) for country-specific quarterly unemployment time series, with the purpose to evaluate the estimated transition functions $F C\left(z_{t-d}\right)$ and $F\left(z_{t-m}^{a}\right)$. When these functions take values close to 1 , the economy can be said to be in a recession, and when they are close to 0 , there is an expansion. Following related empirical work, we set the transition functions at 1 (0) when they exceed (are below) the value of 0.5. This results in pairs of time series, each containing values of 1 and 0 .

To evaluate the business cycle chronologies, indicated by the STAR models for unadjusted data and adjusted data, we use the kappa coefficient introduced in Cohen (1960). This coefficient can be used to evaluate the agreement among the opinions of two observers, in case it cannot be stated that one of the two observers gives the "true" opinion. Define $p_{i j}$ as the proportion of quarters in which $F C\left(z_{t-d}\right)=i$ and $F\left(z_{t-m}^{a}\right)=j$, where $i, j \in\{0,1\}$. The observed proportion of agreement is $p_{00}+p_{11}$. Furthermore, define $m_{0}=p_{00}+p_{10}$, $m_{1}=p_{01}+p_{11}, n_{0}=p_{00}+p_{01}$ and $n_{1}=p_{10}+p_{11}$. The expected agreement is then given by $m_{0} n_{0}+m_{1} n_{1}$. The kappa coefficient is now defined as

$$
k a p p a=\left[p_{00}+p_{11}-\left(m_{0} n_{0}+m_{1} n_{1}\right)\right] /\left[1-\left(m_{0} n_{0}+m_{1} n_{1}\right)\right] .
$$

In case of perfect agreement, $p_{00}+p_{11}=1$, and kappa equals 1 . If there is chance agreement, kappa equals 0 . When kappa is positive, there is agreement, and when it is negative, there is 
disagreement. In order to test whether the estimated kappa differs significantly from 0 , one needs its standard error. Define

$$
\begin{aligned}
& d_{00}=\left[1-\left(m_{0} n_{0}+m_{1} n_{1}\right)\right]-\left[1-\left(p_{00}+p_{11}\right)\right]\left(m_{0}+n_{0}\right) \\
& d_{01}=-\left[1-\left(p_{00}+p_{11}\right)\right]\left(m_{0}+n_{1}\right) \\
& d_{10}=-\left[1-\left(p_{00}+p_{11}\right)\right]\left(m_{1}+n_{0}\right) \\
& d_{11}=\left[1-\left(m_{0} n_{0}+m_{1} n_{1}\right)\right]-\left[1-\left(p_{00}+p_{11}\right)\right]\left(m_{1}+n_{1}\right)
\end{aligned}
$$

and

$$
s=\left(m_{0} n_{0}+m_{1} n_{1}\right)\left(p_{00}+p_{11}\right)-2\left(m_{0} n_{0}+m_{1} n_{1}\right)+\left(p_{00}+p_{11}\right) .
$$

The asymptotic standard error of the kappa coefficient is

$$
\text { ase }=\left\{N\left[1-\left(m_{0} n_{0}+m_{1} n_{1}\right)^{4}\right]\right\}^{-1 / 2}\left\{\left(p_{00} d_{00}^{2}+p_{01} d_{01}^{2}+p_{10} d_{10}^{2}+p_{11} d_{11}^{2}\right)-s^{2}\right\}^{1 / 2},
$$

where $N$ is the number of quarterly observations considered, see Fleiss, Cohen and Everitt (1969) and Schouten (1982). In these studies it is also proved (under general conditions) that kappa/ase asymptotically follows a standard normal distribution.

A second method to evaluate the impact of seasonal adjustment is given by an examination of the estimated peaks and troughs and the implied recessions, given the estimated transition functions $F C\left(z_{t-d}\right)$ and $F\left(z_{t-m}^{a}\right)$. Here, we define a recession as a period where a transition function takes a value of 1 , and which lasts at least 2 quarters.

\section{INTERNATIONAL EVIDENCE}

In this section we consider (SEA)STAR models for sets of quarterly SA and NSA unemployment data. First, we provide some information on the data, and in Section 3.2 we give a discussion of the empirical strategy. Section 3.3 contains the key results.

\subsection{The data}

The data concern 7 industrialized economies, namely the G7 countries. For the US, Canada and France, one can only obtain NSA data on the number of unemployed from the OECD Main Economic Indicators. For the other countries, NSA unemployment rate data are available. The available NSA and SA monthly observations are averaged to quarterly observations. This 
is done for all countries, except for Italy, for which only quarterly data are available. One may question the approach of averaging monthly SA data to quarterly SA data. In fact, a better approach is of course to first create quarterly NSA data, and then to apply adjustment methods. However, an examination of the precise effect of the method pursued on nonlinear modeling is postponed to future research. Finally, the May 1968 observation for France is missing, and we replace it by the average of the May 1967 and May 1969 observations.

For the US, Canada, Germany, France and Italy the quarterly data range from 1960.1 to 1997.3. For the other two countries not as many observations are available. The series for the UK cover 1971.1 to 1997.3 , for Japan the observations start in 1960.1 but end already in 1994.2. Only when the data concern the number of unemployed they are transformed by taking natural logs.

\section{$3.2 \quad$ (SEA)STAR models}

Following the specification strategy outlined in Franses and de Bruin (1999), we find that the SEASTAR model which is generally useful for the NSA data assumes that the delay parameter $d$ takes the value of 1 , and that the number of lags $p$ can be set equal to 5. Additionally, the parameters at lags 2 and 3 , that is $\delta_{2}^{R}, \delta_{3}^{R}, \delta_{2}^{E}$, and $\delta_{3}^{E}$, can be set equal to zero. The transition variable $z_{t}$ is decided to equal

$$
\begin{aligned}
z_{t} & =\left(y_{t}+y_{t-1}+y_{t-2}+y_{t-3}\right)-\left(y_{t-1}+y_{t-2}+y_{t-3}+y_{t-4}\right) \\
& =y_{t}-y_{t-4}
\end{aligned}
$$

which is a variable that is approximately free of seasonality. For the SA data, we will consider $z_{t}^{a}=y_{t}^{a}-y_{t-4}^{a}$. Also, we use AIC to select the value of $k$ (as we are uncertain how seasonal adjustment affects values of $k$ when the order $p$ in a SEASTAR is known.)

\section{- insert Table 1 -}

In the first two columns of Table 1, we present the p-values of the relevant test statistics based on the auxiliary regression in (4). For the UK and Japan we find no evidence for switching regimes. For the US we do not need to include an $F S$ function, and hence 4 seasonal dummies will take care of seasonality. For the other 4 countries, we find evidence in favor of a SEASTAR model as in (1)-(3). 
In the last column of Table 1, we report the p-values of testing for STAR nonlinearity in the SA data. As expected, we find for 5 countries evidence in favor of STAR. For these countries we will fit the model as in (5)-(6).

\section{- insert Table 2 -}

We present some estimation results for the (SEA)STAR models in Table 2. As is well known, the standard error of the $\gamma$ parameter is highly unreliable, and hence these are not reported. We observe that the threshold parameter $\mu$ can take quite distinct values across the SEASTAR and STAR models. Furthermore, we observe that the persistence in the two regimes (measured here by the sum of the AR parameters) tends to be longer for NSA data.

\section{- insert Figures 1 to 5 -}

In Figures 1 to 5, we depict the graphs of the estimated cyclical transition functions $(F C$ and $F$ ) from the SEASTAR and STAR model. Clearly, we can observe that the functions can be different in terms of smoothness and in terms of indicating a cyclical chronology.

\subsection{Is there agreement across chronologies?}

The estimated cyclical transition functions from the STAR and SEASTAR models are used to assign values of "1" (recession) and "0" (expansion), and hence to yield a pair of quarterly time series containing ones and zeroes for each country. In Table 3 in columns 2 to 5 , we give the frequencies (out of the $n$ observations) that the combinations $(i, j)$ occur, with $i$ and $j \in\{0,1\}$. In case of $(1,1)$ and $(0,0)$, the two transition functions display agreement on the business cycle stage.

\section{- insert Table 3 -}

The sixth column of Table 3 displays the estimated agreement $\hat{p}_{11}+\hat{p}_{00}$. It can be seen that this agreement generally is close to 1, with the exception of Italy (agreement is 0.710). The final two columns of Table 3 give the estimated kappa coefficient and its associated asymptotic standard error. Clearly, the kappa values are all significantly positive, and hence there is moderate (Italy) or substantial (other countries) agreement on the business cycle chronologies for nonlinear models for SA and NSA unemployment.

The simulation and empirical results in Franses and Paap (1999) show that recessionary 
periods indicated by Markov switching models for SA data tend to last longer that those found for NSA data. To examine whether their finding carries over to the STAR models, we calculate the average length of recessions implied by both models and present these in Table 4.

\section{- insert Table 4 -}

The results in Table 4 suggest that there can be substantial differences, in the sense that recessions found for SA data tend to last longer, especially for Canada, France and Italy. Comparing the mean and median values of the average recession lengths shows that in general recessions tend to last longer for seasonally adjusted data.

\section{CONCLUSION}

This paper has investigated whether nonlinear time series models for seasonally adjusted or unadjusted unemployment yield different inference on business cycle variation. The analysis was confined to a comparison of implied business cycle chronologies, emerging from (seasonal) smooth transition autoregressive models. The general result is that there is substantial agreement across the chronologies for adjusted and unadjusted data, although the recessions found for adjusted data tend to last longer.

Of course, this paper focused on a set of series, and did not provide any details on specific countries. Since the empirical results show that for none of the countries under study the two models yield the same results, this study may motivate to have a closer look at specific data. It can be interesting in itself to see which quarters are not assigned to a recession in the US for unadjusted data, while they are for adjusted data. Additionally, it is worthwhile to examine the forecasting performance of both models, and to see if recessions can be better predicted by STAR models for unadjusted data or for adjusted data. 
Table 1: Testing for the necessity to include seasonal and/or cyclical transition functions in (SEA)STAR models: p-values of the test statistics

\begin{tabular}{lccc}
\hline \hline & \multicolumn{3}{c}{$\mathrm{p}$-values } \\
\cline { 2 - 4 } Country & $\mathrm{FS}$ & $\mathrm{FC}$ & $\mathrm{F}$ \\
\cline { 2 - 4 } US & 0.691 & 0.071 & 0.068 \\
UK & 0.890 & 0.670 & 0.294 \\
Canada & 0.062 & 0.089 & 0.001 \\
Germany & 0.002 & 0.000 & 0.000 \\
Japan & 0.836 & 0.831 & 0.608 \\
France & 0.014 & 0.026 & 0.006 \\
Italy & 0.001 & 0.024 & 0.019 \\
\hline \hline Note: The test regression is given in (4) with $d=1$.
\end{tabular}


Table 2: Estimated parameters in (SEA)STAR models for (N)SA data (with standard errors in parentheses)

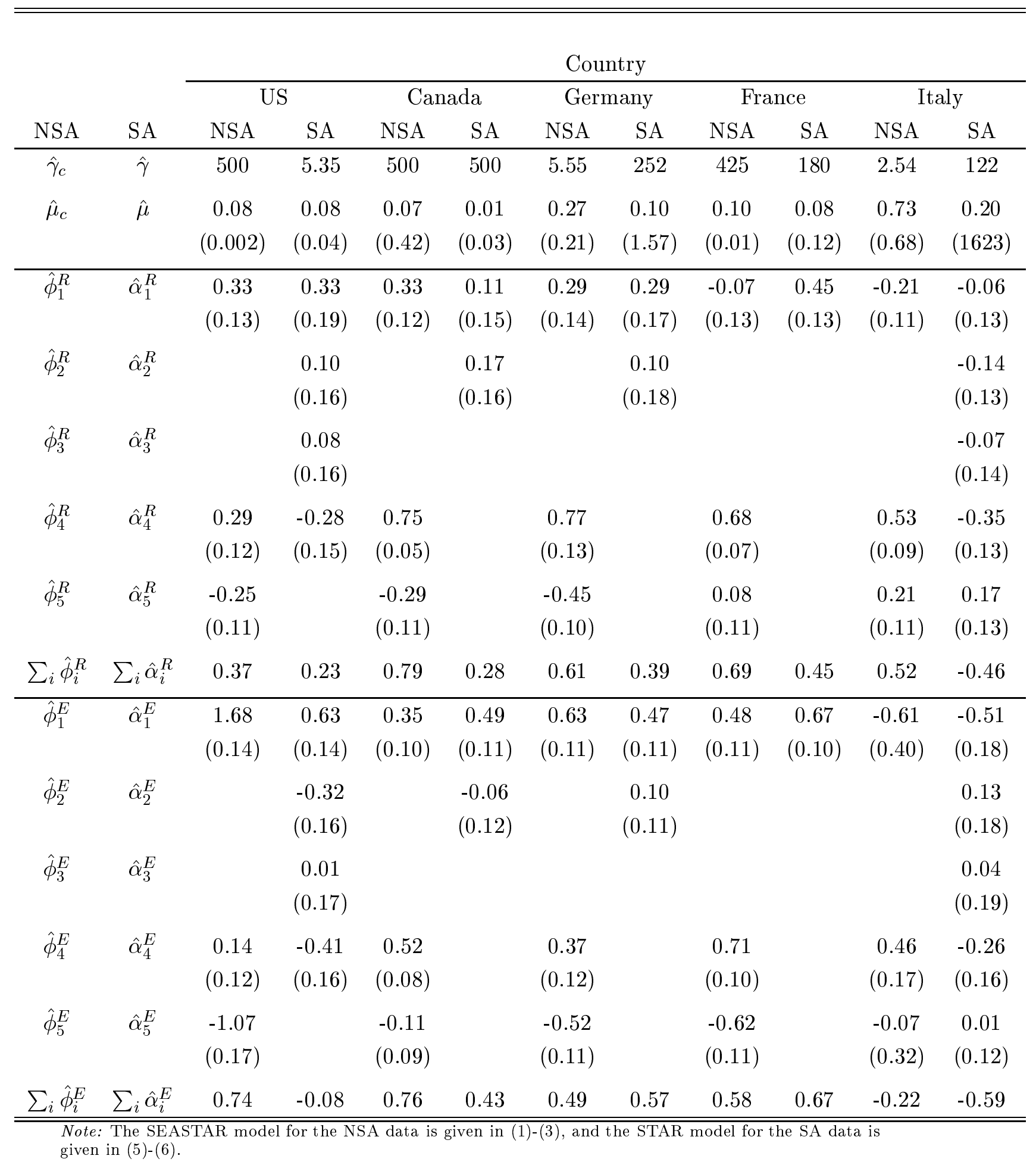


Table 3: Agreement on the nonlinear cycle in unemployment. The cells contain frequencies

\begin{tabular}{lccccccc}
\hline \hline \multicolumn{7}{c}{ States indicated for SA and NSA data, respectively } \\
Country & $(1,1)^{a}$ & $(1,0)$ & $(0,1)$ & $(0,0)$ & Agreement $^{b}$ & Kappa $^{c}$ & $(\text { ase })^{d}$ \\
\hline US & 0.248 & 0.000 & 0.014 & 0.738 & 0.986 & 0.964 & $(0.025)$ \\
Canada & 0.338 & 0.138 & 0.007 & 0.517 & 0.855 & 0.706 & $(0.057)$ \\
Germany & 0.338 & 0.076 & 0.000 & 0.586 & 0.924 & 0.839 & $(0.046)$ \\
France & 0.372 & 0.083 & 0.000 & 0.545 & 0.917 & 0.831 & $(0.046)$ \\
Italy & 0.228 & 0.290 & 0.000 & 0.483 & 0.710 & 0.431 & $(0.061)$ \\
Average & 0.305 & 0.117 & 0.004 & 0.574 & 0.878 & 0.754 & \\
\hline \hline
\end{tabular}


Table 4: Average length of "recessions" in unemployment, where a recession is defined as any observation for which the switching function in the (SEA)STAR model takes a value $>0.5$ (with number of "recessionary" periods in parentheses)

\begin{tabular}{lcccc}
\hline \hline & & & & \\
Country & SEASTAR for NSA data & STAR for SA data \\
\hline US & 6.0 & $(6)$ & 6.6 & $(5)$ \\
Canada & 6.6 & $(7)$ & 8.3 & $(8)$ \\
Germany & 7.8 & $(6)$ & 7.5 & $(8)$ \\
France & 5.4 & $(10)$ & 6.6 & $(10)$ \\
Italy & 3.0 & $(8)$ & 7.2 & $(10)$ \\
Mean & 5.8 & & 7.2 & \\
Median & 6.0 & & 7.2 & \\
\hline \hline
\end{tabular}


US: FC SEASTAR over time

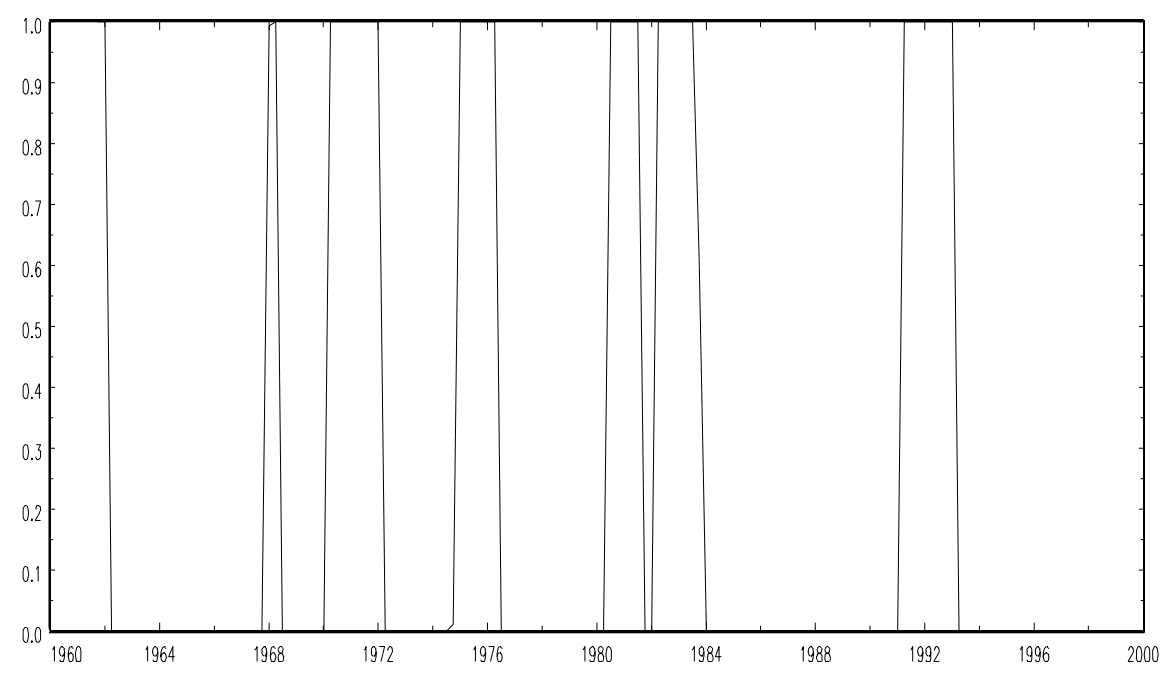

US: F STAR over time

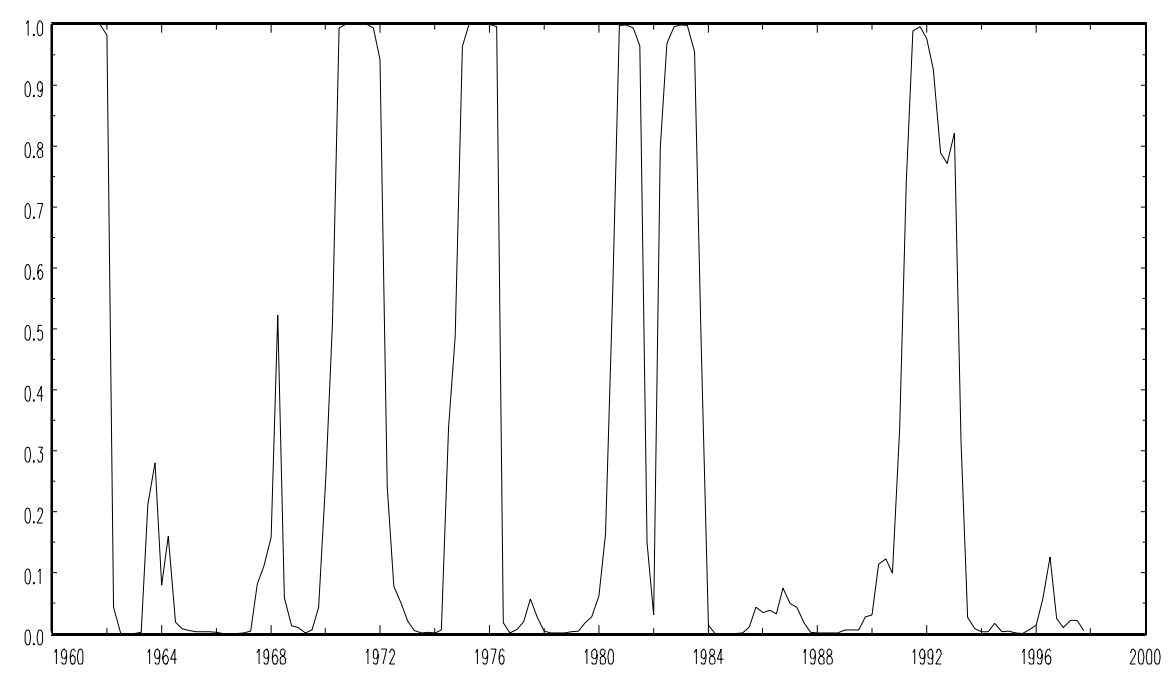

Figure 1: The estimated business cycle transition function from the SEASTAR model (top panel) and the STAR model (bottom panel): the US 
CANADA: FC SEASTAR over time

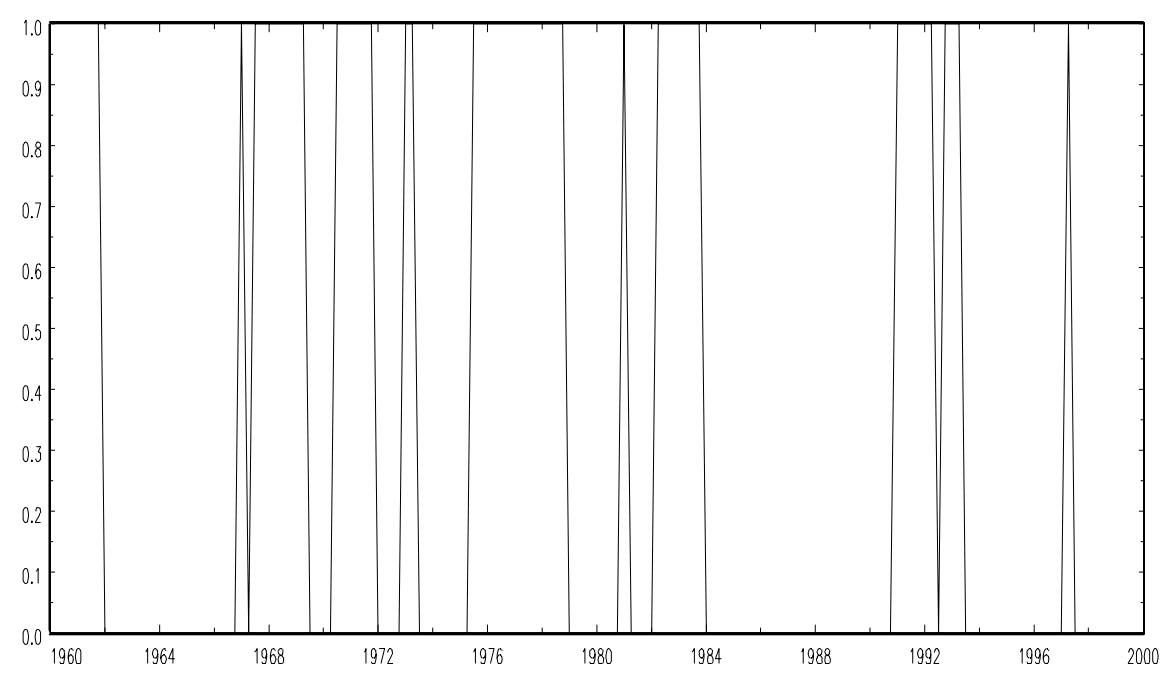

CANADA: F STAR over time

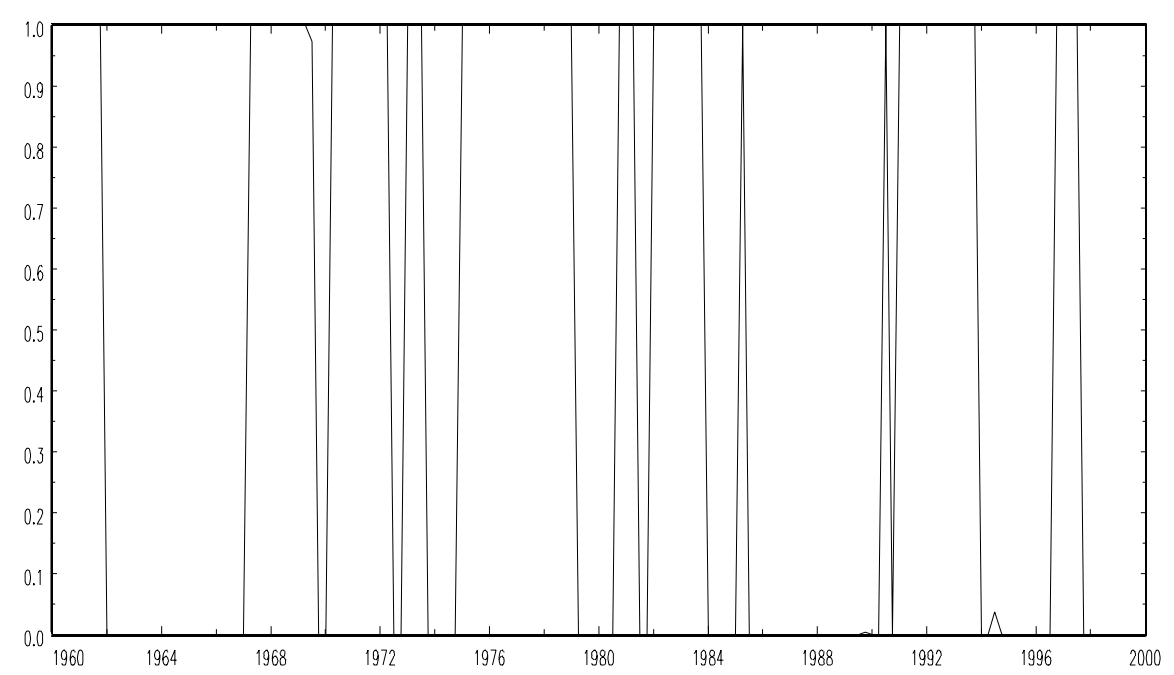

Figure 2: The estimated business cycle transition function from the SEASTAR model (top panel) and the STAR model (bottom panel): Canada 


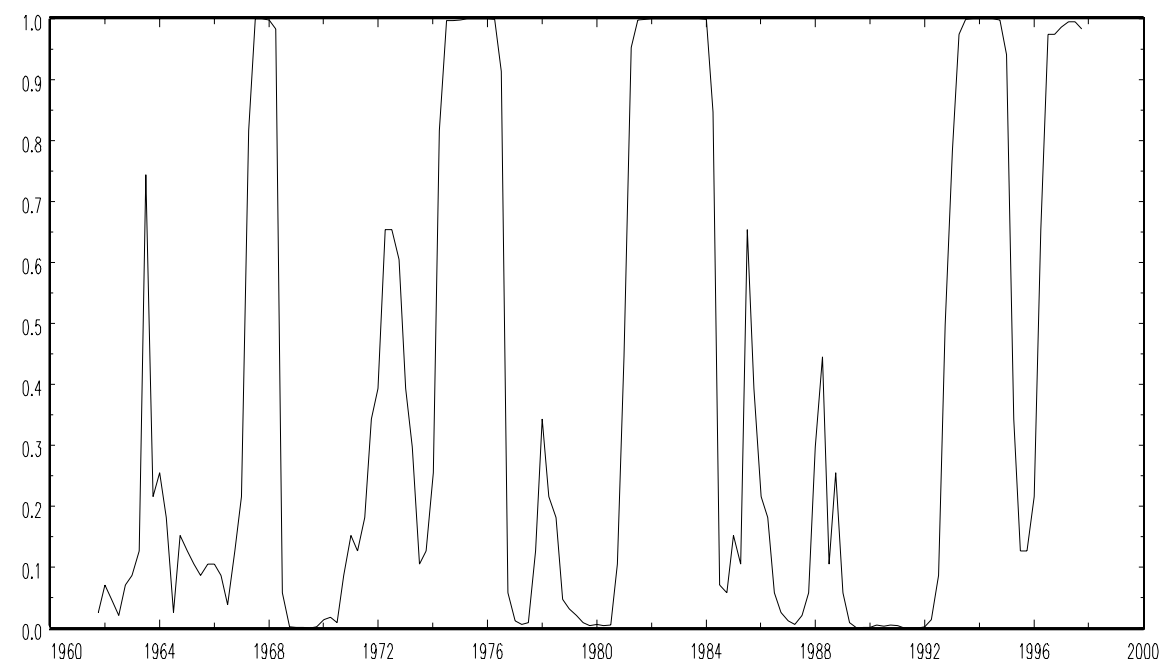

GERMANY: F STAR over time

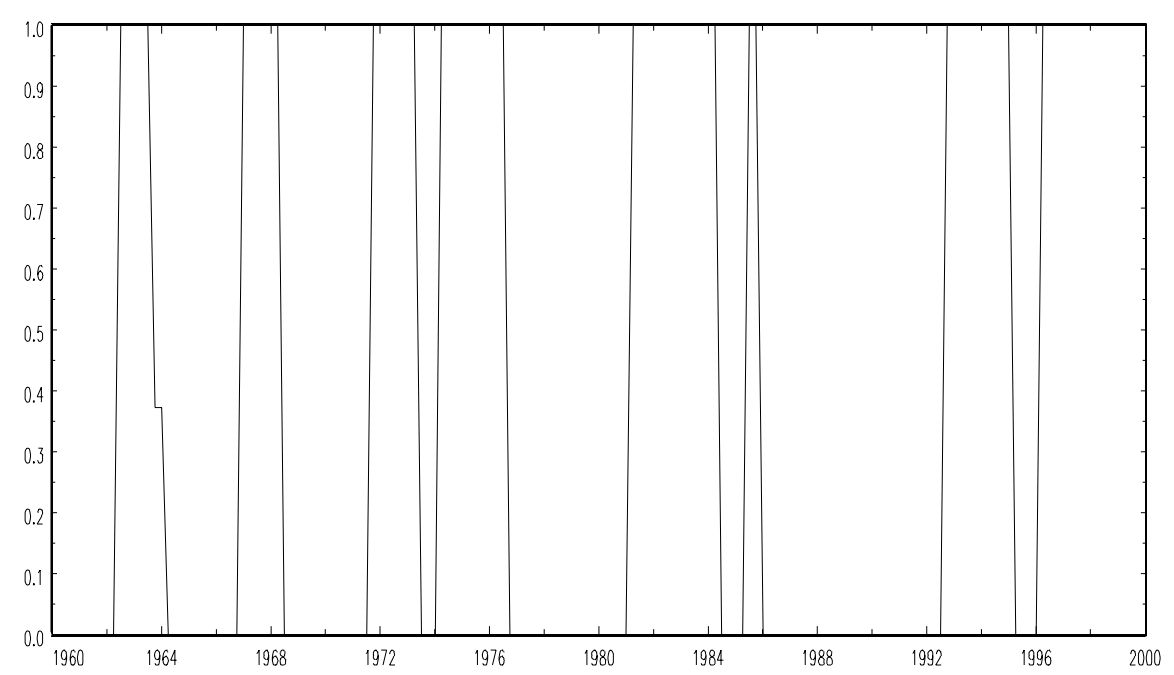

Figure 3: The estimated business cycle transition function from the SEASTAR model (top panel) and the STAR model (bottom panel): Germany 
FRANCE: FC SEASTAR over time

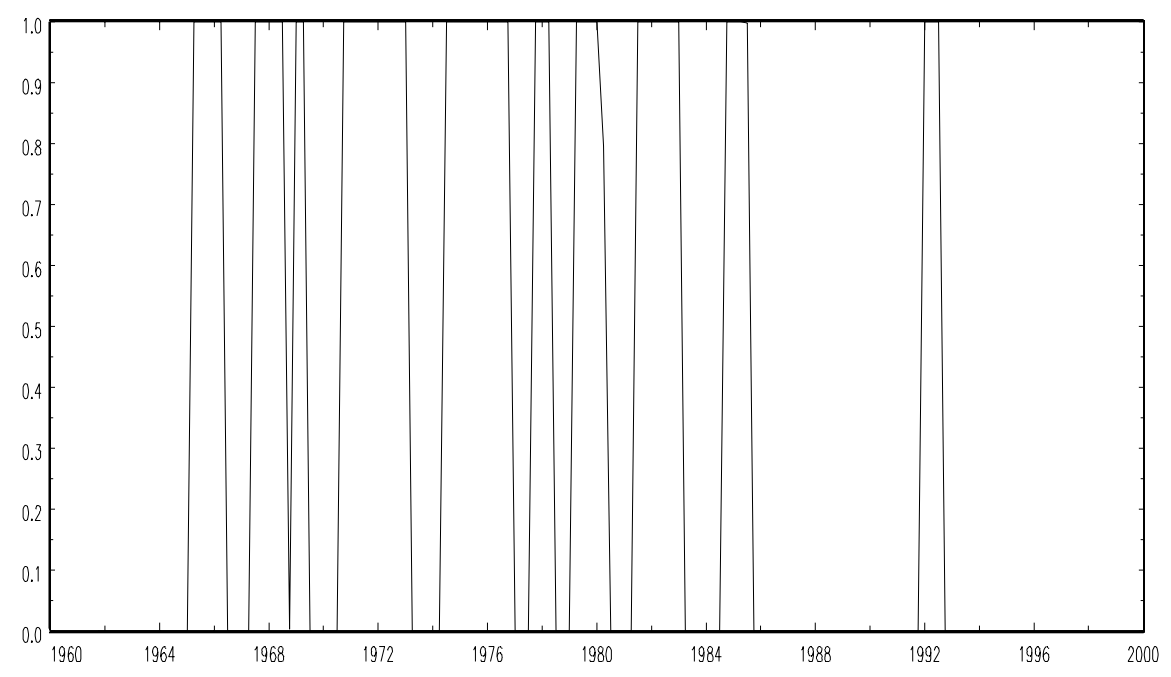

FRANCE: F STAR over time

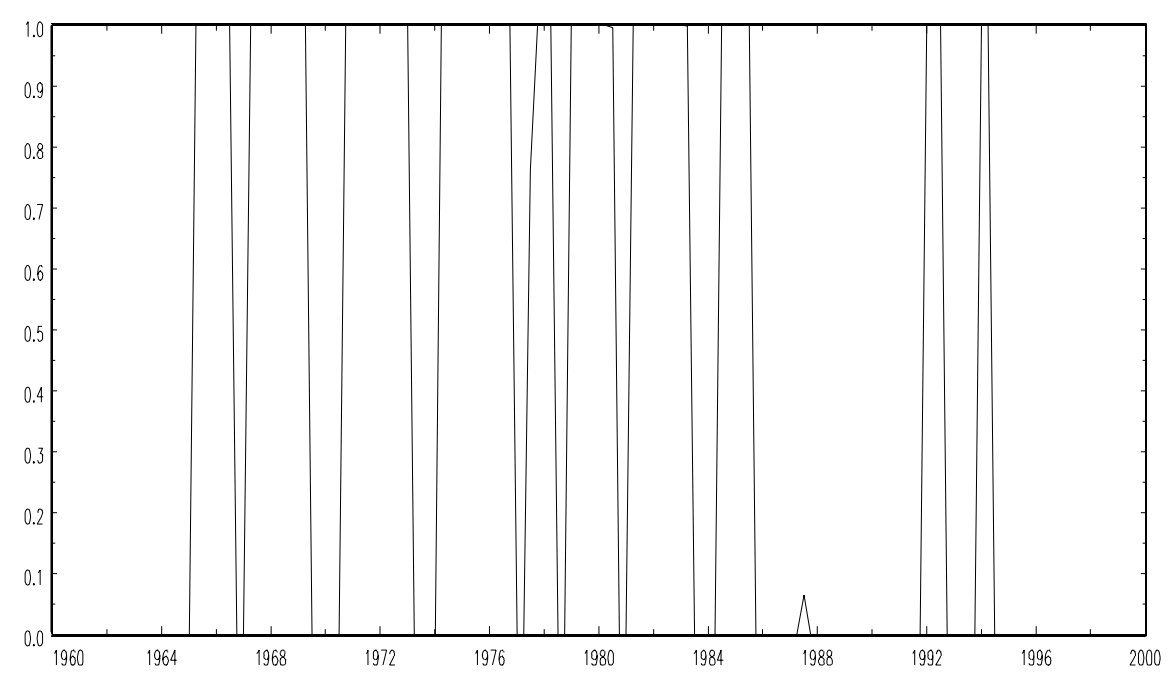

Figure 4: The estimated business cycle transition function from the SEASTAR model (top panel) and the STAR model (bottom panel): France 
ITALY: FC SEASTAR over time

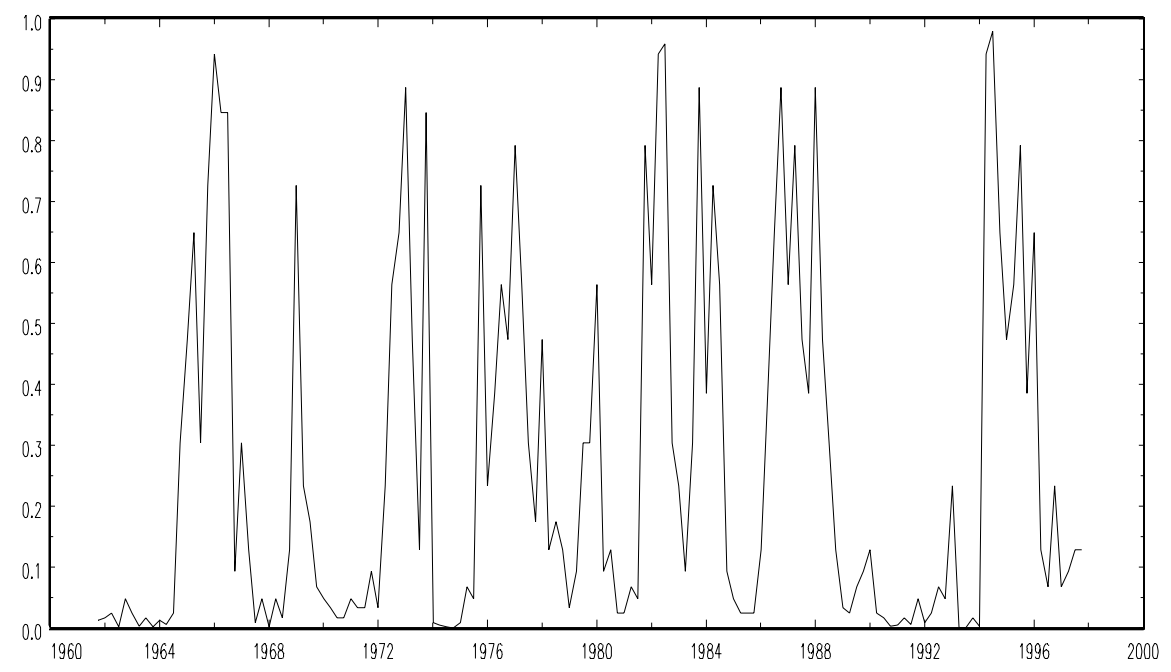

ITALY: F STAR over time

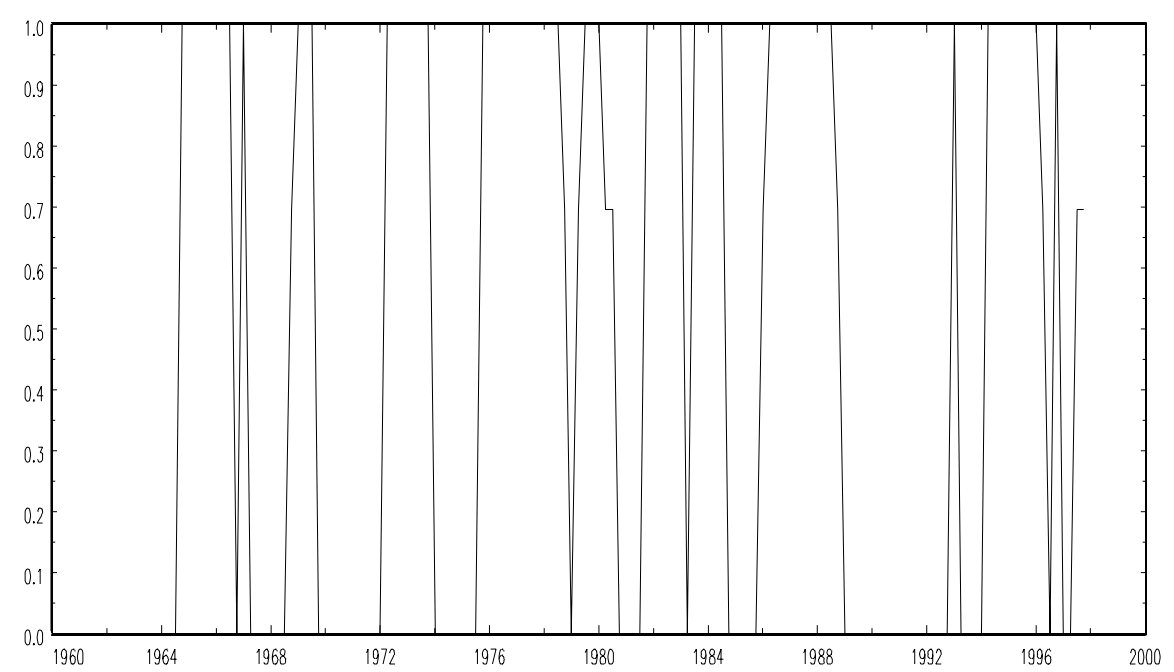

Figure 5: The estimated business cycle transition function from the SEASTAR model (top panel) and the STAR model (bottom panel): Italy 


\section{References}

[1] Canova, F. and E. Ghysels (1994), Changes in Seasonal Patterns: Are they Cyclical?, Journal of Economic Dynamics and Control, 18, 1143-1171.

[2] Cohen, J. (1960), A Coefficient of Agreement for Nominal Scales, Educational and Psychological Measurement, 20, 37-46.

[3] Fleiss, J.L., J. Cohen and B.S. Everitt (1969), Large Sample Standard Errors of Kappa and Weighted Kappa, Psychological Bulletin, 72, 323-327.

[4] Franses, P.H. (1995), Quarterly U.S. Unemployment: Cycles, Seasons and Asymmetries, Empirical Economics, 20, 717-725.

[5] Franses, P.H. (1998), Does Seasonality in Unemployment Change with its (Nonlinear) Cycle?, Econometric Institute Report 9809, Erasmus University Rotterdam.

[6] Franses, P.H. and P. de Bruin (1999), Seasonal Smooth Transition Autoregression, Unpublished Manuscript, Econometric Institute, Erasmus University Rotterdam.

[7] Franses, P.H. and R. Paap (1999), Does Seasonality Influence the Dating of Business Cycle Turning Points?, Journal of Macroeconomics, 21, 79-92.

[8] Ghysels, E. and P. Perron (1993), The Effects of Linear Filters on Dynamic Time Series with Structural Change, Journal of Econometrics, 70, 69-98.

[9] Granger, C.W.J. and T. Teräsvirta (1993), Modeling Nonlinear Economic Relationships, Oxford: Oxford University Press.

[10] Miron, J.A. (1996), The Economics of Seasonal Cycles, Cambridge US: MIT Press.

[11] Ooms, M. and P.H. Franses (1997), On Periodic Correlations Between Estimated Seasonal and Nonseasonal Components for US and German Unemployment, Journal of Business and Economic Statistics, 15, 470-481.

[12] Schouten, H.J.A. (1982), Measuring Pairwise Interobserved Agreement when All Subjects are Judged by the Same Observers, Statistica Neerlandica, 36, 45-61. 